\title{
Innovative and Assistive eHealth Technologies for Smart Therapeutic and Rehabilitation Outdoor Spaces for the Elderly Demographic
}

\author{
Bruno Marques ${ }^{1, *}$, Jacqueline McIntosh ${ }^{1}$, Alvin Valera ${ }^{2}$ and Anuroop Gaddam ${ }^{3}$ \\ 1 School of Architecture, Victoria University of Wellington, 6140 Wellington, New Zealand; \\ jacqueline.mcintosh@vuw.ac.nz \\ 2 School of Engineering and Computer Sciences, Victoria University of Wellington, \\ 6140 Wellington, New Zealand; alvin.valera@vuw.ac.nz \\ 3 School of Information Technology, Deakin University, 3125 Victoria, Australia; \\ anuroop.gaddam@deakin.edu.au \\ * Correspondence: bruno.marques@vuw.ac.nz; Tel.: +64-4-463-4718
}

Received: 27 August 2020; Accepted: 21 October 2020; Published: 22 October 2020

\begin{abstract}
The use of technology for social connectivity and achieving engagement goals is increasingly essential to the overall well-being of our rapidly ageing population. While much of the extant literature has focused on home automation and indoor remote health monitoring; there is a growing literature that finds personal health and overall well-being improves when physical activities are conducted outdoors. This study presents a review of possible innovative and assistive eHealth technologies suitable for smart therapeutic and rehabilitation outdoor spaces for older persons. The article also presents key performance metrics required of eHealth technologies to ensure robust, timely and reliable biometric data transfer between patients in a therapeutic landscape environment and respective medical centres. A literature review of relevant publications with a primary focus of integrating sensors and eHealth technologies in outdoor spaces to collect and transfer data from the elderly demographic who engage such built landscapes to appropriate stakeholders was conducted. A content analysis was carried out to synthesize outcomes of the literature review. The study finds that research in assistive eHealth technologies and interfaces for outdoor therapeutic spaces is in its nascent stages and has limited generalisability. The level of technology uptake and readiness for smart outdoor spaces is still developing and is currently being outpaced by the growth of elderly fitness zones in public spaces. Further research is needed to explore those eHealth technologies with interactive feedback mechanisms that are suitable for outdoor therapeutic environments.
\end{abstract}

Keywords: eHealth technologies; elderly people; remote health monitoring; mobile health; therapeutic landscape; eHealth architecture

\section{Introduction}

The ageing global population of people aged 65 years or more is expected to grow to 1.5 billion people by 2050 [1]. As the accompanying increased propensity for impairment is expected to outpace healthcare services, a new paradigm shift in health product services and processes is required. Advances in medical science coupled with healthier ageing have meant that people are living longer and for the first time, one kind of illness is replaced by another [2]. Impairments are increasingly associated with molecular and cellular damage over time as physical and mental capacity dwindles with a growing risk of health issues such as dementia, hearing loss, poor vision and other cognitive disorders. This demographic change presents a critical challenge in terms of cost of health care 
services and consultation burden on the medical personnel [3] and the need to move from reactive to preventive health care delivery has become inevitable [4].

The mismatch between the supply and demand for care and health professionals highlights the need for health care services to transit into a system where data and technology can be accessed to make this shift a reality. Well-organised, reliable, timely and valid biometric data collected remotely and linked with a health information system can provide tremendous help for evidence-based decision making, monitoring, evaluation and proper health management of the elderly [2,5]. The development of a smart platform for integrating biometric data with health services through eHealth and eCare technologies is now essential to managing clinical reporting and auditing. Information and communication technology (ICT) now has a major role to play in determining who provides care, where, what, how and when services are provided [6].

The use of ICT in health and health-related fields aims to enhance the quality, efficiency and effectiveness of health-service management. Scholz [7] highlights four applications of eHealth solutions, they include:

1. Personalized homecare services, telemedicine and mobile health (mHealth): this include smart watches for daily activities monitoring, remote wearable patient monitoring devices, teleconsultations and eReferrals.

2. Integrated health information systems: such as ePrescription, electronic health records (EHR) and eDispensing.

3. Non-clinical systems: such as online support groups, online health information services and patient flow management systems.

4. Clinical systems: these are mainly used by health professionals within or outside health care institutions such as computer-assisted diagnostics, medication management systems and pharmacy information systems.

Merilampi and Sirkka [6] presented seven partially overlapping groupings of eHealth technologies, they are:

i. Assistive technologies: these are tools, equipment and devices used to help an individual in overall management of daily life and to compensate sensory, cognitive and physical impairments. Advanced equipment now includes environmental management devices, sensors, embedded systems and videophone network.

ii. Safety and social technologies: safety technologies are various security units networked with other assistance and surveillance services such as smart floors, bed occupancy sensors, safety bracelets and other integrated sensors interconnected to a remote center for processing. A typical example of a social technology is the health TV which provides speech and visual links through a broadband connection. Big data handling, false alarms and privacy issues are concerns in adopting safety and social technologies.

iii. Health technologies: these are self-monitoring and self-care systems such as blood pressure monitors, eHealth portals and other internet-based services with remote connection with health professionals.

iv. Self-activation and personal development technologies: these can be referred to as sport technologies designed to support, analyze and monitor individuals' development and performance. Examples are pedometers, accelerometers, smart sport watches and activity bracelets. Another recent development under this application is gamification, where motivation is provided for repetitive rehabilitation or exercise.

v. Design-for-all and ambient assisted living (AAL) technologies: the design-for-all concept provides equal opportunities for everyone irrespective of age, gender, ability to function and cultural orientation. A typical example is the sliding door which shows all the design-for-all criteria such as sustainability and affordability. However, this type of eHealth technology application might not always be suitable for all and cost-effective; for example, prosthesis usage requires customization. AAL technologies provides elder care to promote well-being and 
independence. Examples include robots, medication optimization equipment and wearable technologies.

vi. Gerontechnology: This is a general term and includes technology from all the previously mentioned categories. Its overall aim is to develop age-friendly technologies in order to promote independence, well-being and prevent age-related health problems.

vii. Hospital technology and EHR systems: these are patient systems used to integrate data from wearables, smart home-type technologies, electronic patients and health records for monitoring health progress of patients. They provide useful health information such as physical assessment, referral and daily charting used in health and social care.

This era is currently witnessing a strong collaboration across disciplines and research fields such as therapeutic landscape design, medicine, engineering and information technology for health care applications and market opportunities. This ongoing collaboration has triggered a lot of research interest due to its potential socio-economic impacts [8]. The eHealth outlook extends over various perspectives such as the technological, political, economic, international cooperation, research and stakeholders [7]. Furthermore, eHealth technologies are not just limited to digital systems but include other technologies such as robots and other automated systems for performing predictive processes and advanced analytics for supplying new insights into intricate health issues [5].

It is well-known that personal well-being and health status improve when older people participate in physical activity especially in outdoor spaces [9]. The World Health Organization encourages the creation and use of outdoor spaces in a way that makes the elderly demographic physically engage with their environment [2]. The development of age-specific therapeutic outdoor spaces continues to rise in most parts of the world in an attempt to reduce age-related loss of function, impairment and disability among older persons [10]. Consequently, studies are currently required to review the application of assistive technologies in a therapeutic outdoor environment [11]. There is a need to develop a smart platform for collecting and integrating biometric data with EHR services via eHealth technologies suitable for such landscapes.

Apart from the general benefits of physical well-being, outdoor interactive exercises in recreation parks and public places by elderly people have other advantages such as the psychological benefits of belonging and social engagement [12,13]. Active ageing exercises help to train motor and balance skills necessary for the confidence and independence needed in performing daily activities [12]. Interaction with outdoor spaces provide both sensory and non-sensory benefits which have been found to reduce the effects of dementia and depression. While the sensory benefits of being outdoors have been well studied, there is an increased interest in the non-sensory benefits through the ingestion or inhalation of phytoncides, negative ions in the air and microbes [9].

In response to demands for health care interventions for the elderly in public spaces, there is a growing proliferation of "elderly fitness zones" in parks and reserves around the world [14]. However, ongoing use by the elderly population is uncommon and much of the equipment is not fit for purpose [15]. Research is required to improve both the integration and uptake of outdoor equipment and furniture use with public spaces. Effective and efficient measurement technologies and medical data are needed to allow the user to obtain feedback and monitor their progress through the acquisition and utilization of biometric data from these outdoor spaces.

This paper explores the potential of technologies suitable for outdoor environments to assist individuals affected by age related loss of function, impairment and disability and includes the evaluation of a platform for integrating biometric data with health services through eHealth technologies. It presents a detail review of eHealth technology and interface requirements to ensure a robust, timely, reliable and valid biometric data exchange between health professionals or centres and patients in outdoor interactive spaces. It examines the current smart interactive technologies and the necessary elements of outdoor spaces and performance elements to provide support for pre-frail older persons and their corresponding medical personnel in detection, diagnosis and rehabilitation support in a therapeutic landscape environment. Interactive feedback technologies within outdoor spaces to ensure close monitoring and evidence-based decision making are highlighted with their key performance metrics. 


\section{Materials and Methods}

\subsection{Data Sources, Search Strategy and Study Selection}

An electronic search of Scopus, Google Scholar, Institute of Electrical and Electronic Engineers (IEEE) Xplore and Association Computing Machinery (ACM) Digital Library databases was conducted with data range from January 2005 to April 2018 using the Preferred Reporting Items for Systematic Reviews and Meta-Analyses (PRISMA) statement [16]. In order to identify the relevant literature, alternate terms such as "eHealth", "outdoor spaces", "assistive technologies", "therapeutic landscape", "elderly", "old people", "smart spaces", "smart outdoor", "mHealth", "eHealth technologies", "outdoor environment", "ambient assistive living", "aged", "rehabilitation", were used with Boolean operators (e.g., AND, OR) to determine the solution space. Data sources generated a total of 2975 results from all the databases and passed through multi-level screening as shown in Figure 1.

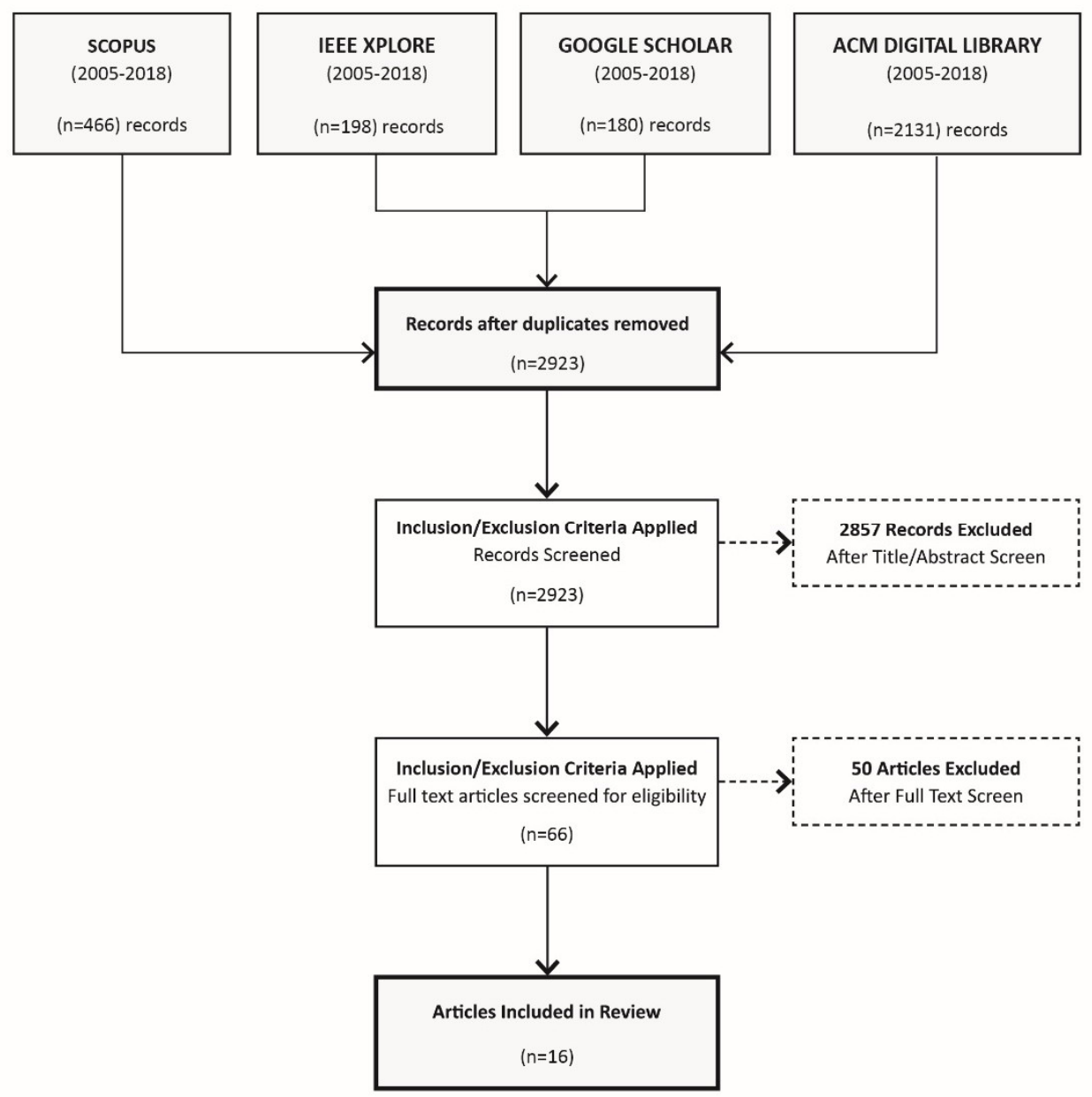

Figure 1. Literature search and assessment using the Preferred Reporting Items for Systematic Reviews and Meta-Analyses (PRISMA) flow diagram.

\subsection{Inclusion and Exclusion Criteria}

After removing duplicates (52), the remaining articles were screened using the inclusion and exclusion criteria as shown in Table 1 . These conditions were developed and applied to appropriately scope this review article in line with predetermined objectives. 
Table 1. Study inclusion and exclusion criteria.

\begin{tabular}{|c|c|}
\hline Inclusion Criteria & Exclusion Criteria \\
\hline $\begin{array}{c}\text { Peer reviewed articles (2005-2018) } \\
\text { Empirical research } \\
\text { English language literature } \\
\text { Outdoor built environment } \\
\text { eHealth/assistive technologies in outdoor space } \\
\text { Aged } 60 \text { years or older with complex needs }\end{array}$ & $\begin{array}{l}\text { Non-peer reviewed articles } \\
\text { Smart homes and home-health monitoring } \\
\text { Conference proceedings } \\
\text { Narrative reviews, lecture notes and studies } \\
\text { published in theses } \\
\text { Non-health related studies and technology } \\
\text { applications }\end{array}$ \\
\hline
\end{tabular}

The outdoor built environment encompasses places and spaces created or modified by people including buildings, parks, and transportation systems. In recent years, public health research has expanded the definition of built environment to include healthy food access, community gardens, walkability and bikeability [17]. For the purposes of this paper, eHealth has been defined as an emerging field which brings together medical informatics, public health and business, and primarily refers to health services and information delivered or enhanced through the Internet and related technologies. The term characterizes not only technical development, but also a state-of-mind, a way of thinking, an attitude, and a commitment to networked, global thinking, to improve health care locally, regionally, and worldwide by using information and eHealth technology [18].

\subsection{Study Selection}

After the initial application of the inclusion and exclusion criteria with "Title/Abstract" screening, 2857 articles were excluded and 66 included. Articles were considered relevant if their abstracts showed that the study provided an insight into the application of technologies for remote monitoring of the elderly demographic within an outdoor environment. When the relevance of a paper was difficult to ascertain after reading the abstract, then the full text was read to decide whether to include it or not. In the final analysis, after full text screening only 16 articles remained, which have been summarized and are presented in this review. In addition, to reduce publication bias a variety of databases were included which helps to ensure a more thorough search and achieve greater levels of sensitivity.

\section{Results}

\subsection{Assistive eHealth Technologies and Interfaces for Outdoor Interactive Spaces}

For health management, evidence-based decision-making, monitoring and assessment of health status of the elderly demographic, reliable and robust eHealth technologies are important in providing timely health status information of patients [2]. A smart and interactive platform is required to integrate valid biometric data collected in outdoor spaces with health services via reliable eHealth technologies. The availability of outdoor space and the increasing demand for health information now make such platforms useful in providing interactive feedback to help older persons affected by morbidity, impairment and age-related loss of function. Effective measurement and eHealth technologies with medical data capture requirements are pivotal in ensuring collection, protection and utilization of biometric data.

Ambient intelligence and computational techniques (algorithms) are basic requirements for building a smart open space to support older persons in an outdoor environment. Doukas et al. [11] identified three key areas in building such a landscape, which are:

1. The use of onsite or remote monitoring tools for rapid detection of environmental changes, changing needs and events. Examples of tools are mobile and static sensors, and software tools for collection, fusion and analysis of data.

2. Risk detection and alerts to trigger activation of assistive devices for an emergency response.

3. Localization of affected older persons with easy to use communication interfaces. 
Network sensors, radio frequency badges, Global Positioning System (GPS) and terrestrial infrastructures (cell ID of a subscribed mobile terminal) are examples of location-based assistive health technologies [11]. Biometric data from smart devices such as smartphones, smart glasses and smartwatches with GPS-processing using compatible applications, such as MyVigi and iWander $[19,20]$. Foxlin [21] developed a system known as NavShoe for position-tracking based on inertial sensing. The system used a shoe-mounted miniature magnetometer coupled wirelessly with a radio frequency (RF) module to a personal digital assistant (PDA) to track patients. Abdel-Aziz et al. [22] identified four information and communication technology (ICT) elements that could play major roles in public spaces. They include Wi-Fi networks, digital interactive media facades, and interactive public displays and smartphones applications. These factors determine the planning and design of outdoor spaces to satisfy users' needs and provide support for remote monitoring.

Lin et al. [23] presented a system which integrates technologies of radio frequency identification, Global Positioning System (GPS), a Global System for Mobile Communications (GSM) network and geographic information system (GIS), as shown in Figure 2, to construct a stray prevention network for older people with dementia without interfering with their daily lives.

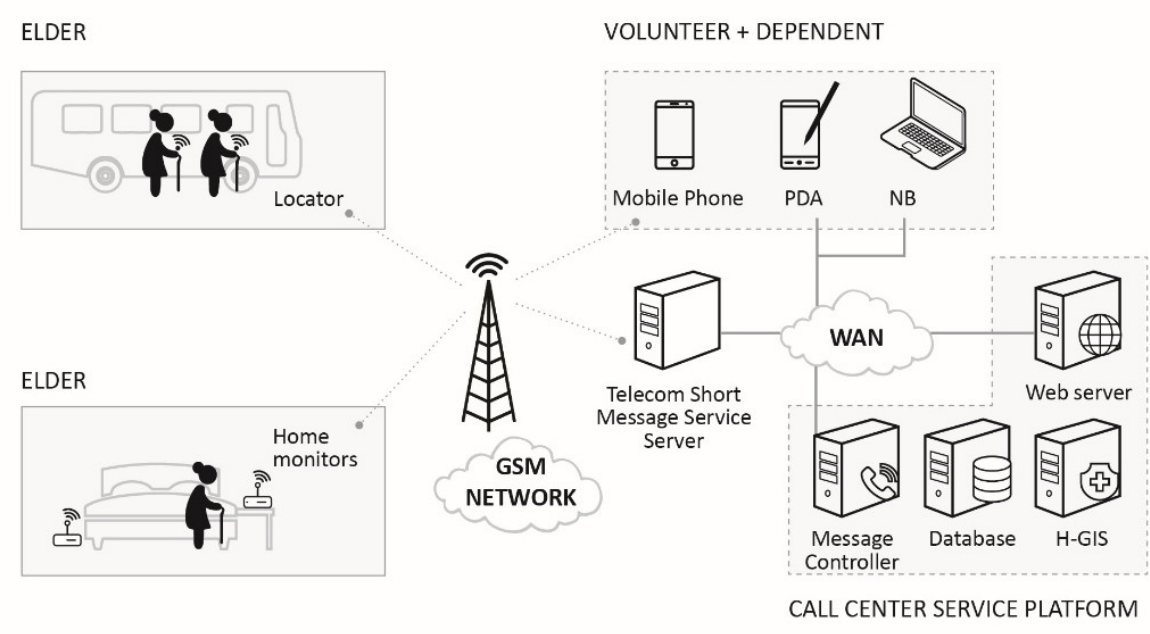

Figure 2. Service health management care system architecture. (Adapted from Lin et al. [23]).

The system comprises a web service server (for portal service), a database server (for data storage and management), a message controller server, and a health-GIS server (for intervention). Caregivers or medical centres locate the real-time positions of the affected patient via mobile phone, PDA, Notebook PC, and various mobile devices through the service platform. In 2008, Lin et al. [24] integrated radio frequency identification (RFID) technology to the development of an indoor and outdoor active safety monitoring system for dementia patients. The system is capable of sending messages automatically to caregivers whenever an elderly person approaches a risky zone or strays too far away. Also, the mechanism uses different size tags and tame transformation signatures (TTS) algorithm to encrypt tag IDs for privacy. Similarly, Rainham et al. [25] developed and tested a lightweight, wearable GPS receiver to measure the temporal and spatial features of daily activities, with the capability of logging location information for up to 70 hours before recharging.

In 2010, Oswald et al. [26] presented the use of tracking technologies utilising a wrist-watch with body contact sensors, RF and GPS units over a GSM network for the analysis of outdoor mobility of dementia patients. However, the accuracy of data collected varied depending on the local terrain and weather conditions. Also in 2010, Zheng et al. [27] developed a personalised self-management system to provide support for self-management of chronic conditions with a decision support system (DSS) to identify abnormal activity and differentiate life-style patterns. The proposed system used a smart phone with integrated accelerometer and GPS modules to monitor outdoor activity, providing both location and activity-based information. 
In a European Union (EU)-funded project for older persons with multiple medical conditions, Boulos et al. [28] presented the use of a smartphone app coupled with the GPS location sensor to initiate a bidirectional communication between the patient's personal area network (PAN) and a remote server that is accessible by medical personnel over the internet. Similarly, in addressing a cardiac rehabilitation condition, Worringham et al. [29] presented an eHealth network consisting of a smartphone, electrocardiograph (ECG) and GPS-based system for remotely monitoring the exercise of patients. The system provided a more flexible way to remotely carry out unsupervised cardiac rehabilitation via a programmed smartphone to a secure server for real-time monitoring by qualified medical personnel. During exercise sessions, the smartphone streamed general packet radio service (GPRS) data to a secure server (Figure 3) enabling the ECG trace, heart rate, walking speed, elapsed distance and patient location to be viewed in real-time. Other studies related to the use of GPS-based systems report that such technology associated with smart devices not only enables the location of patients during an emergency more efficiently but also reduces the time in monitoring and evaluation a patient [30,31]. GPS-based tracking systems can enhance location and mobility awareness, which in turn, assists healthcare providers in detecting adverse health conditions and to quickly act upon them.

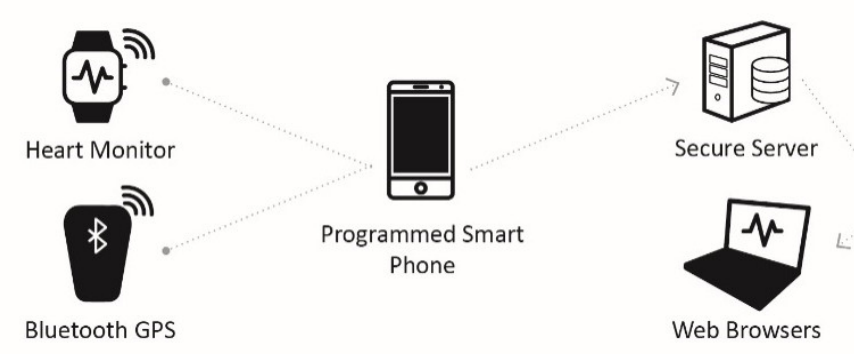

Figure 3. Components of the remote monitoring system. (Adapted from Worringham et al. [29]).

Heikkilä et al. [32] modelled an intelligent furniture network which could be used in an outdoor space for tracking residents' posture and detection of abnormal living patterns using a very low-cost low-intrusive capacitive proximity sensors based on Institute of Electrical and Electronics Engineers (IEEE) 802.15.4 Medium Access Control (MAC) and IEEE 802.15.4a hardware layer. In the proposed architecture, the sensors were integrated into the furniture which include a chair, a sofa and a bed, and then connected to a microcontroller based wireless sensor network (WSN) master node and a gateway as depicted in Figure 4. A universal asynchronous receiver/transmitter (Universal Asynchronous Receiver-Transmitter, COM/UART) serial line was used to connect the master node and gateway, linked to the database and user services with fixed or wireless Internet Protocol (IP) network with or without 802.15.4/IP conversion.

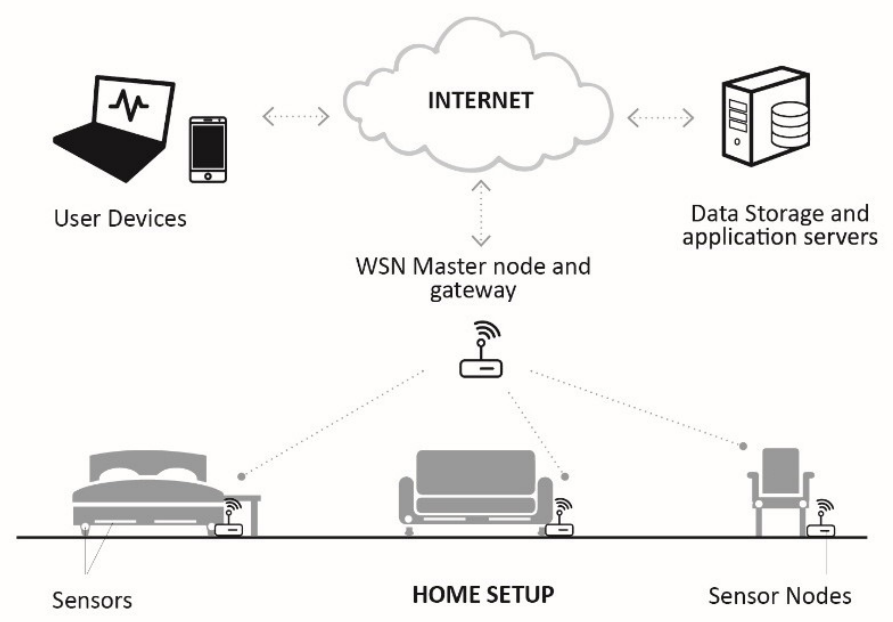


Figure 4. Intelligent furniture network connected to Internet based services. (Adapted from Heikkilä et al. [32]).

Sensors such as accelerometers and gyroscopes integrated with wearable devices are used for data acquisition and then passed though different algorithms to detect the fall. The proposed framework is also extended for wandering risk and mHealth solutions. Interested readers are referred to Schwickert et al. [33] for a comprehensive review on sensors for fall detection.

Yared et al. [34] proposed a schema for detecting falls in an outdoor environment using wearables such as smartphones and sensors as shown in Figure 5. They propose the use of eHealth technologies capable of delivering ubiquitous assistance services and integrated platforms that support safety, independence and overall well-being of older persons in outdoor spaces. Islam et al. [35] applied Internet of Things (IoT) technology to health care delivery and services. IoT is defined as a technological concept of showing a connected set of anything, anyone, anyplace any service and any network. Although IoT is in its infancy in medical applications, it is projected to revolutionise and redesign modern health care with promising techno-economic and social aspects. Islam et al. [35] surveyed the advances in IoT-based health care technologies, state-of-the-art network architectures and industrial trends.

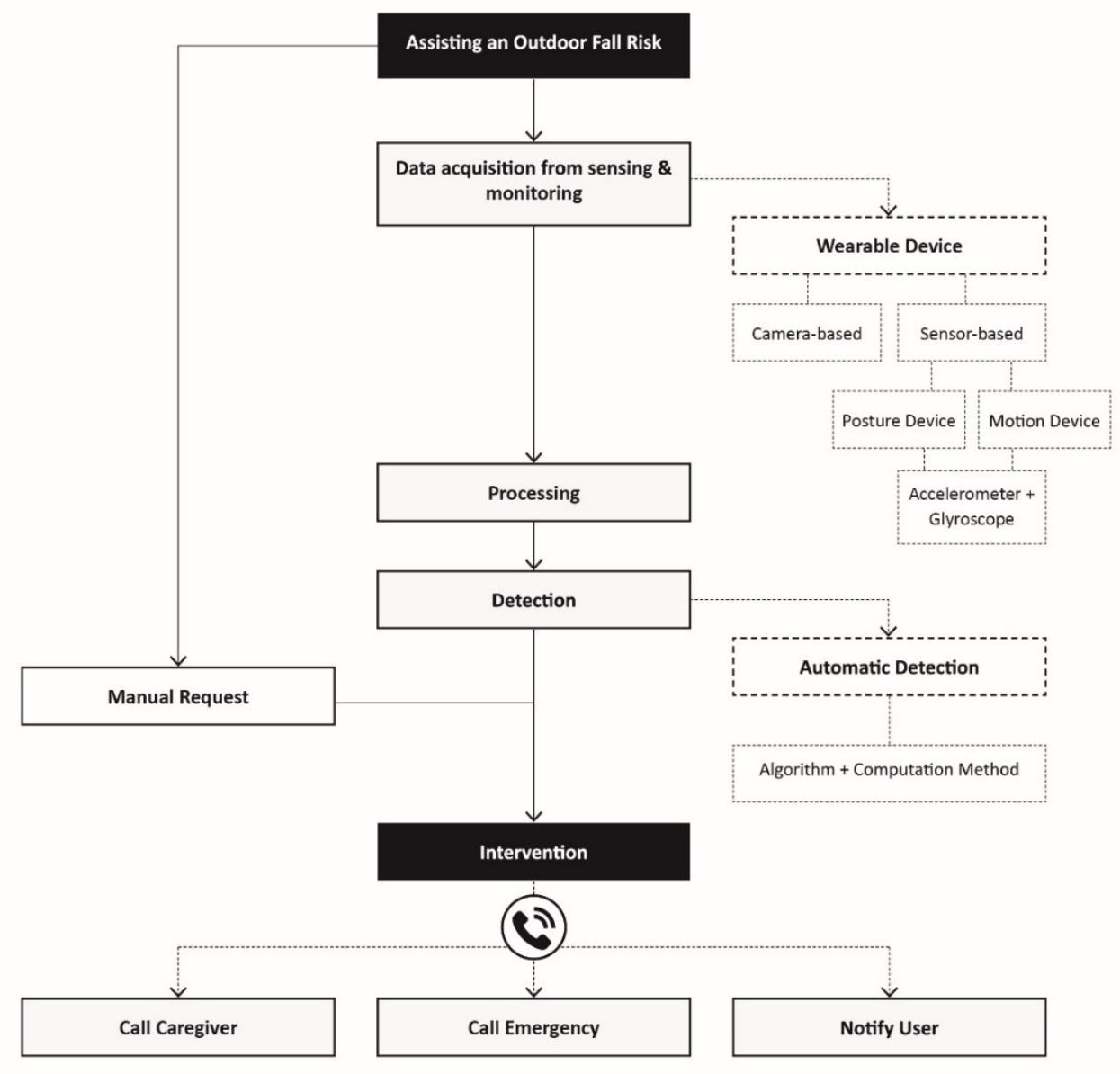

Figure 5. Assistive technology framework for fall risk. (Adapted from Yared et al. [34]).

A smart outdoor eHealth monitoring device to detect fall and ECG signals for older persons with a proposed multi-thread method in order to improve both the response time and detection accuracy was proposed by Wang et al. [36]. The proposed intelligent system consists of a multi-function healthcare box to be carried around by the elderly patient, monitoring the GPS location and ECG data of the patient as shown in Figure 6. This research also found that the accuracy of detection was dependent on movement, location variation and interference. 


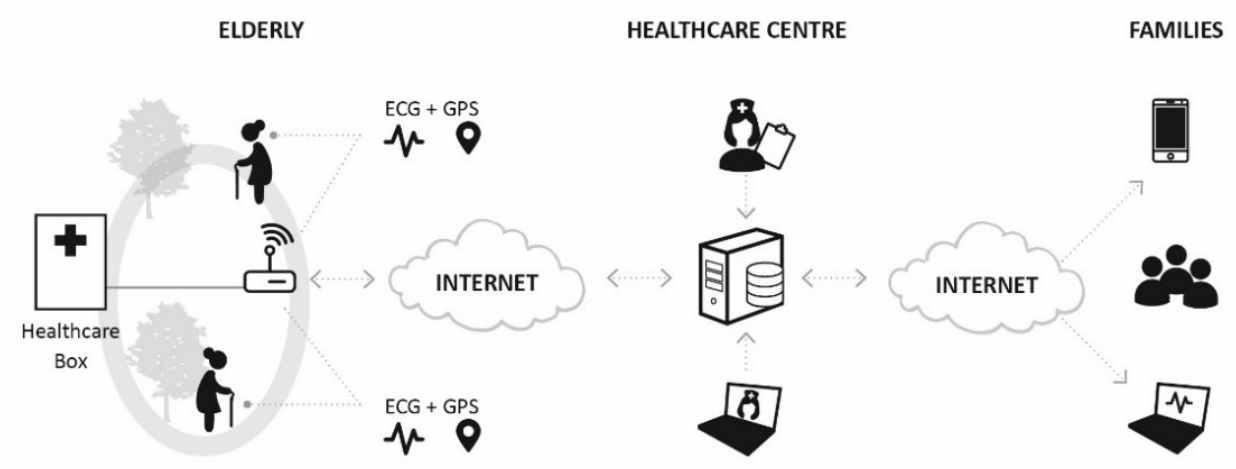

Figure 6. An outdoor intelligent architecture of the proposed healthcare system. (Adapted from Wang et al. [36]).

In 2016, Hossain and Muhammad [37] presented an IoT-enabled eHealth monitoring framework, where integrated devices such as mobile devices and sensors collected ECG and other healthcare data to be securely sent to the Cloud for seamless access by medical personnel. These data were watermarked for security reasons and then sent via communication media such as Bluetooth, $\mathrm{Wi}-\mathrm{Fi}$, internet and cellular technologies. Another IoT-aware architecture for performing behavioural analysis and risk detection of elderly patients over cellular technology used a different type of sensor to capture mobility, outdoor localization and ambient parameters [38]. The proposed smart cityoriented system captured and processed heterogeneous data in an assistive environment at multiple levels. The data model allowed participating cities to choose the level of abstraction that could be used for system integration [38]. Similarly, Hu et al. [39] used IoT medical sensors through Cloud computing to monitor chronic health conditions of elderly patients over cellular technology. The proposed Cloud computing architecture can safely and securely monitor the health of older persons for chronic conditions, anywhere including outdoor locations.

Garcia et al. [40] proposed the "Safe Neighbourhood" approach which combined data from multiple sources with collective intelligence over merged technologies such as mobile, ambient, and artificial intelligence technologies. Data obtained from the elderly via sensors integrated with a mobile phone were sent through the internet to the eHealth monitor. Trusted, private and neighbourhood-based social networks such as "Nextdoor" and "GoNeighbour" were adopted for data privacy and security.

Finally, Chan et al. [41] implemented an indoor/outdoor smart system with multisensory and wearable units to monitor older person's movement trajectories. Functions such as mobility, posture assessment, fall detection and prevention, were monitored using a combination of technologies which included:

- eWatch for activity recognition and integrating activity data with location information.

- $\quad$ GPS positioning system to locate the individual in outdoor scenario.

- RFID-based location-finding and tracking with guidance system.

- GSM for patient's alert message and communication.

Table 2 provides a summary of the reviewed articles with highlights on the medical conditions being addressed, main outcomes, and acquisition and data eHealth technologies.

Table 2. Medical conditions, acquisition technologies, data communication technologies and main outcomes.

\begin{tabular}{ccccc}
\hline Study & $\begin{array}{c}\text { Medical } \\
\text { Condition and } \\
\text { Disability } \\
\text { Addressed }\end{array}$ & $\begin{array}{c}\text { Acquisition of } \\
\text { Technology }\end{array}$ & $\begin{array}{c}\text { Data } \\
\text { Communication } \\
\text { Technology }\end{array}$ & Main Outcomes \\
\hline Foxlin [20] & $\begin{array}{c}\text { Patients' daily } \\
\text { activities }\end{array}$ & $\begin{array}{c}\text { wireless inertial sensor } \\
\text { (magnetometer) with }\end{array}$ & $\begin{array}{c}\text { Radio frequency (RF) } \\
\text { module }\end{array}$ & $\begin{array}{c}\text { The system used a shoe- } \\
\text { mounted miniature }\end{array}$ \\
\hline
\end{tabular}




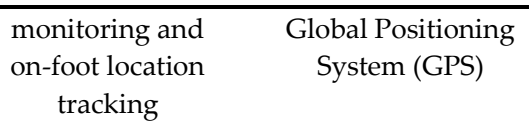

Lin et al. [23]

Dementia

Disease assessment and outdoor safety monitoring of dementia patients

Rainham et al. [25]

Monitoring location

Chronic conditions Zheng et al. (diabetes [27] mellitus and cardiovascular disease) Analysis of

Oswald et al. outdoor mobility [26] in the face of dementia

Chronic conditions

Boulos et al [28] (diabetes mellitus and cardiovascular disease)

Worringham et al. [29]

Cardiac rehabilitation

Heikkilä et al. Posture and [32] activity monitoring

Monitoring and

Yared et al.

[34] detecting falls, wandering and other related risks
Motion sensor with GPS

Global System for Mobile

Communications (GSM)

RFID tag

Accelerometer and GPS

Wrist-watch with body contact sensor and RF capability, and GPS

smart garment with wireless health sensors and GPS

Electrocardiogram (ECG), heart rate and GPS modules

Cellular technology and Bluetooth

Fixed or wireless Internet Protocol (IP) network and Institute of Electrical and

Electronics Engineers (IEEE) 802.15.4

Accelerometer, gyroscope, Radiofrequency identification (RFID) tags and GPS
Cellular technology magnetometer coupled

wirelessly to a personal digital assistant (PDA) to track patients.

Emergency messages and commands from remote server were sent via a GSM link.

The system used different size tags and tame transformation signatures (TTS) algorithm to ensure privacy.

A lightweight, wearable GPS receiver to measure the temporal and spatial features of daily activities was developed.

A smart phone having an accelerometer and a GPS was used to monitor patients' outdoor activity and location.

The accuracy of data collected varied depending on the local terrain and weather conditions.

A smartphone app coupled with the GPS location sensor to initiate a bidirectional communication between the patient's PAN and a remote server accessible over the internet was developed.

The system provided a more flexible way to remotely monitor supervised cardiac rehabilitation via a programmed smartphone to a

secure server for real-time monitoring by qualified medical personnel.

Intelligent furniture was developed to detect variations in activities and postures using a proprietary WSN and internet.

The use of eHealth technologies capable of delivering ubiquitous assistance services and integrated platforms that support safety, independence and overall well-being of older persons in outdoor spaces was proposed. 


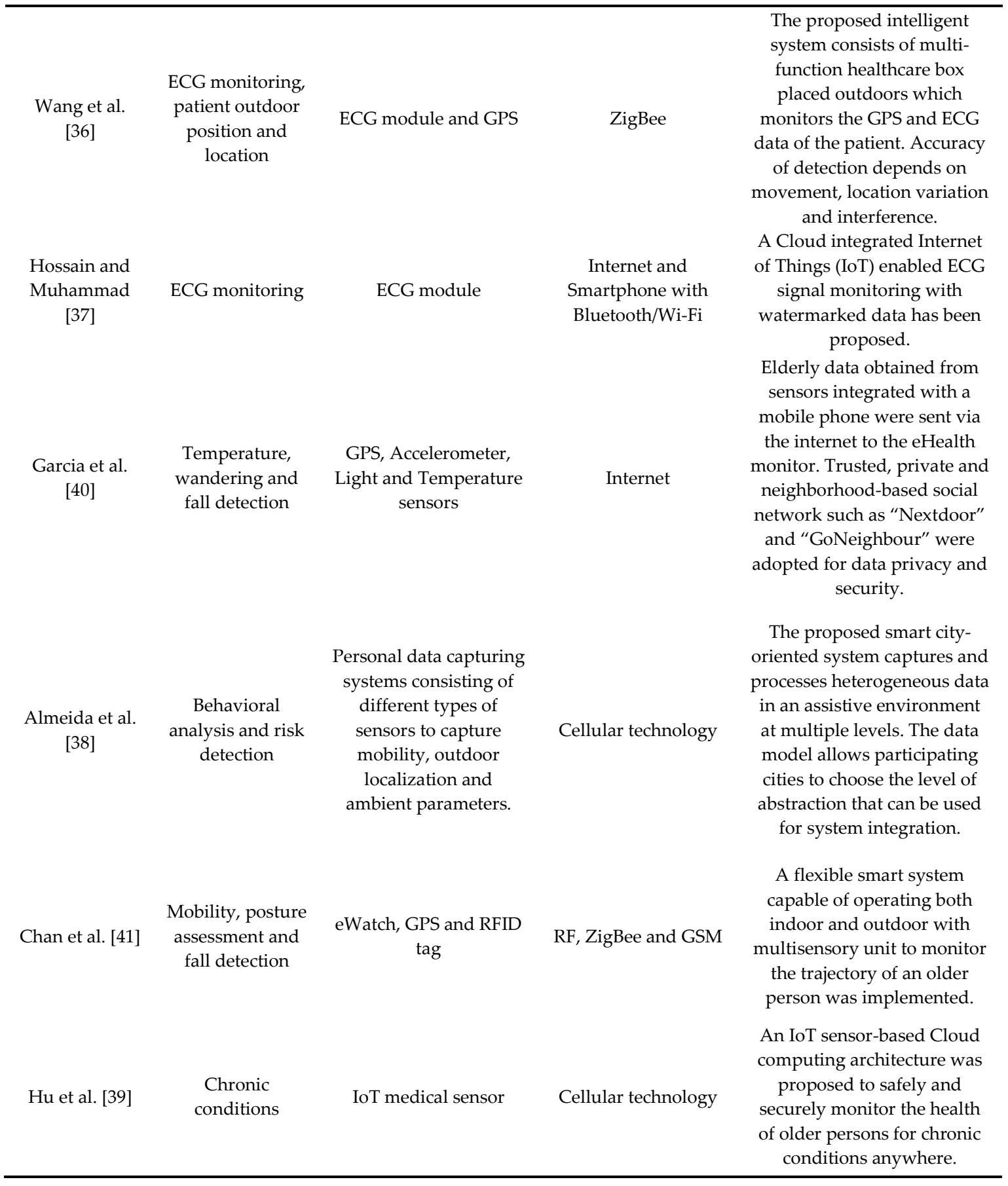

\subsection{Mobile Health (mHealth)}

The emergence and revolution of smartphone technology is a key milestone of the last decade with myriads of applications and unprecedented opportunities in almost every sphere of society. The rapid uptake of the mobile technology now offers a new platform to provide actionable mobile medical devices [42]. This is evidenced by the proliferation of a large number of medical and healthrelated apps, numbering about 40,000 in 2017 [42].

As mobile technology becomes increasingly pervasive, another component of eHealth, referred to as mobile health (mHealth), has emerged. The main benefits of mHealth are penetration into population groups and subgroups, availability of a wide variety of apps, anytime and anywhere connectivity, and personalised data capture for individual users [43]. Approximately 3.4 billion people are estimated to own a smartphone by 2018, which implies that mobile technology with integrated well-being applications is pivotal to healthcare transformation [7]. The use of global positioning systems (GPS) and location-enabled smartphones continues to grow and provide a 
platform for a wide range of applications such as remote health monitoring for assisted and independent living [28,44]. In general, technology-driven location-based services depend on underlying networks such as fixed line access, cell based and GPS assisted services. However, for each case the reliability, accuracy, efficiency and speed of service delivery cannot be compromised, especially in cases of emergency and rescue intervention [45].

The European Telecommunication Standards Institute (ETSI) provides parameterization of telecommunication services for eHealth applications. These include the following considerations and are summarised in Table 3:

- Ubiquity: a technology is referred to as ubiquitous when it is omnipresent within the zone of its deployment.

- Mobility: this refers to the capability of a device to modify its physical connection point to the telecommunication network without losing its logical connectivity.

- Security: this includes service authorization, confidentiality provision, and station and infrastructure authentication.

- Connection capability: this can be circuit or packet mode of operation. An example of a circuit mode is the conventional telephony with a continuous electrical connection between end points with orderly arrangement of data at the point of exit. The packet mode is currently in use with discrete packets via different routes and can arrive out of order.

- Connection topology: this could be point to point (unicast), point to multipoint (multicast) or point to all points (broadcast).

- Content type: examples include data, video, audio and image.

- Quality of Service (QoS): this refers to the ability of a device to maintain an established connection.

Table 3. Summary of wide area communication technologies for eHealth applications [45].

\begin{tabular}{|c|c|c|c|c|c|c|}
\hline & $\begin{array}{c}\text { Global System for } \\
\text { Mobile } \\
\text { Communications } \\
\text { (GSM) }\end{array}$ & $\begin{array}{c}\text { General } \\
\text { Packet } \\
\text { Radio } \\
\text { Services } \\
\text { (GPRS) } \\
\end{array}$ & $\begin{array}{c}\text { Terrestrial } \\
\text { Trunked } \\
\text { Radio } \\
\text { (TETRA) }\end{array}$ & $\begin{array}{c}\text { Third } \\
\text { Generation } \\
(3 G)\end{array}$ & $\begin{array}{c}\text { Wireless } \\
\text { Fidelity (WiFi) } \\
\text { (IEEE } \\
\text { 802.11a/b/g/n) }\end{array}$ & $\begin{array}{c}\text { Worldwide } \\
\text { Interoperability } \\
\text { for Microwave } \\
\text { Access (WiMAX) }\end{array}$ \\
\hline Description & $\begin{array}{l}\text { Cellular network } \\
\text { used for mobile } \\
\text { phones. }\end{array}$ & $\begin{array}{l}\text { General } \\
\text { Packet } \\
\text { Radio } \\
\text { Service }\end{array}$ & $\begin{array}{l}\text { Private } \\
\text { mobile } \\
\text { radio for } \\
\text { ambulance } \\
\text { and a host } \\
\text { of other } \\
\text { services }\end{array}$ & $\begin{array}{l}\text { Third- } \\
\text { generation } \\
\text { mobile } \\
\text { phone } \\
\text { network } \\
\text { technology }\end{array}$ & $\begin{array}{l}\text { A suite of } \\
\text { wireless } \\
\text { Ethernet } \\
\text { standards. }\end{array}$ & $\begin{array}{l}\text { Worldwide } \\
\text { Interoperability } \\
\text { for Microwave } \\
\text { Access operating } \\
\text { in the licensed } \\
\text { spectrum zone. }\end{array}$ \\
\hline Mobility & Yes & Yes & Yes & Yes & Limited & Yes \\
\hline Security & Privacy enhanced & $\begin{array}{c}\text { Privacy } \\
\text { enhanced }\end{array}$ & Yes & $\begin{array}{c}\text { Privacy } \\
\text { enhanced }\end{array}$ & No & No \\
\hline $\begin{array}{l}\text { Connection } \\
\text { capability }\end{array}$ & Packet, Circuit & Packet & $\begin{array}{l}\text { Packet, } \\
\text { Circuit }\end{array}$ & $\begin{array}{l}\text { Packet, } \\
\text { Circuit }\end{array}$ & Packet & Packet \\
\hline $\begin{array}{c}\text { Connection } \\
\text { topology }\end{array}$ & Unicast & Unicast & $\begin{array}{l}\text { Unicast, } \\
\text { Multicast, } \\
\text { Broadcast }\end{array}$ & $\begin{array}{l}\text { Unicast, } \\
\text { Multicast, } \\
\text { Broadcast }\end{array}$ & $\begin{array}{l}\text { Unicast, } \\
\text { Multicast, } \\
\text { Broadcast }\end{array}$ & $\begin{array}{c}\text { Unicast, Multicast, } \\
\text { Broadcast }\end{array}$ \\
\hline Content & Audio & Data & Audio, Data & $\begin{array}{c}\text { Audio, Data, } \\
\text { Video }\end{array}$ & Data & Data, Video \\
\hline
\end{tabular}

\subsection{Reference Architecture for eHealth}

There is a growing global trend towards electronic health records (EHR) [46-48]. Three considerations for eHealth communication architecture are critical. These include reliability, security and accuracy [49]. However, eHealth communication technologies are also expected to be userfriendly, ubiquitous and user-centric in order to maximize their benefit [7]. In addition, overall 
persistence for events and occasional temporal persistence for sensor data stream represent two key requirements for architectural development for eHealth monitoring [32].

The Continua Health Alliance is a non-profit standardization industry and a multi-stakeholder group which provides a framework for creating interoperable personal health devices and solutions. The Continua Health Alliance in providing a reference architecture has made significant progress in developing the pioneer blueprint of an interoperable end-to-end device for local area network (LAN) and wide area network (WAN) interfaces [50]. Figure 7 shows the Continua personal telehealth ecosystem indicating device connectivity with various enterprise services. Continua's primary aim is to build a system of personal health solutions that are interoperable by certifying and branding Continua-enabled products to support disease management, ageing independently, health and fitness [51].

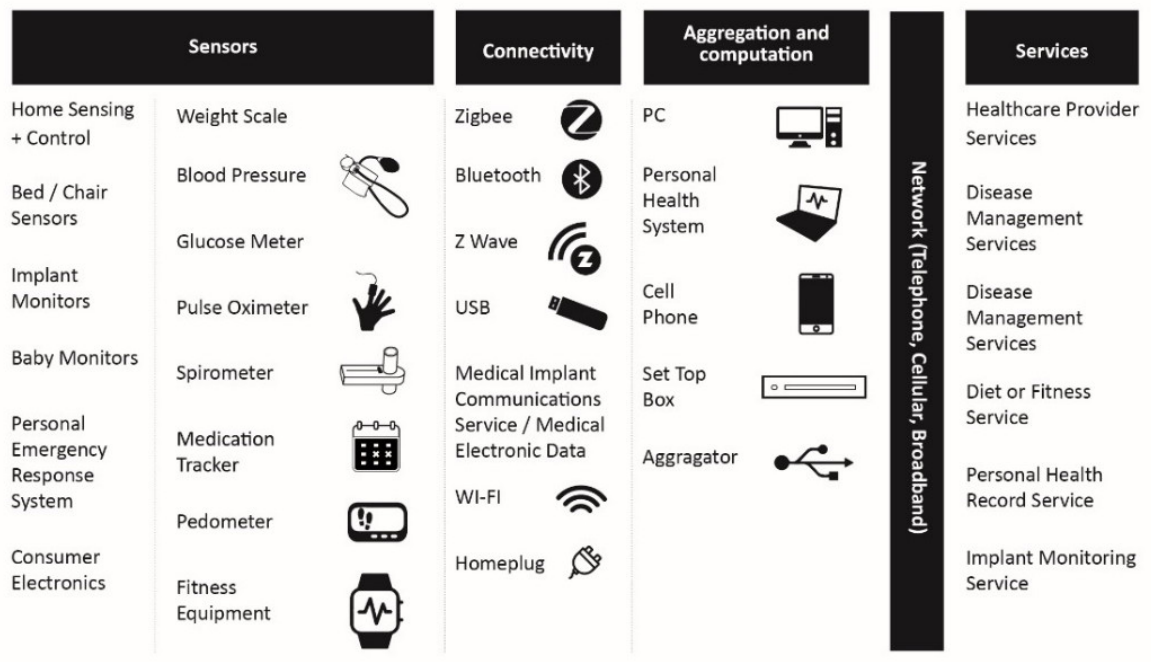

Figure 7. Continua personal eHealth ecosystem. (Adapted from Carroll et al. [52]).

The PAN interface connects the PAN device (a sensor or an actuator) to the application-hosting device (cellphone or a personal computer) via wired or wireless technologies [50,52]. Figure 8 shows the hierarchical Continua open systems interconnection (OSI) 7-layer model with relevant standards for the PAN interface. The PAN lower layers consist of the classic OSI layers 1-4 with USB and Bluetooth as communication technology options while the upper layers comprise the standard OSI layers 5-7 which are deployed using the ISO/IEEE Std 11073-20601 Optimized Data Exchange Protocol. 


\begin{tabular}{|c|c|c|}
\hline & \multicolumn{2}{|c|}{$\begin{array}{l}\text { Device Specialization Layer } \\
\text { ISO / IEEE Std 11073-104ZZ }\end{array}$} \\
\hline & $\begin{array}{l}\text { Pulse Oximeter } \\
\qquad-10404\end{array}$ & $\begin{array}{c}\text { Thermometer } \\
-10408\end{array}$ \\
\hline & $\begin{array}{l}\text { Weighting Scale } \\
\quad-10415\end{array}$ & $\begin{array}{l}\text { Strength, Fitness and } \\
\text { Activity - } 10442\end{array}$ \\
\hline & $\begin{array}{l}\text { Blood pressure } \\
-10407\end{array}$ & $\begin{array}{l}\text { Glucose Meter } \\
-10417\end{array}$ \\
\hline & $\begin{array}{c}\text { Independent Living } \\
\text { Activity Hub } \\
-10471\end{array}$ & $\begin{array}{l}\text { CV Fitness } \\
\text { and Activity } \\
-10441\end{array}$ \\
\hline $\begin{array}{l}\text { Higher - Layer } \\
\text { Protocols 5-7 }\end{array}$ & \multicolumn{2}{|c|}{$\begin{array}{l}\text { Optimized Data Exchange Protocol Layer } \\
\text { ISO / IEEE Std 11073-20601 }\end{array}$} \\
\hline \multirow[t]{2}{*}{$\begin{array}{l}\text { Lower - Layer } \\
\text { Protocols 1-4 }\end{array}$} & \multicolumn{2}{|c|}{ Transport Layer } \\
\hline & $\begin{array}{l}\text { Bluetooth } \\
\text { Health } \\
\text { Device Profile }\end{array}$ & $\begin{array}{l}\text { USB Personal } \\
\text { Healthcare } \\
\text { Device Class }\end{array}$ \\
\hline
\end{tabular}

Figure 8. The Continua open systems interconnection (OSI) 7-layer construct with respective standards (Adapted from Carroll et al. [52] and Moorman [51]).

In addition, the Continua Health Alliance provides an end-to-end reference architecture showing the different network tiers, device topology and constraints as depicted in Figure 9.

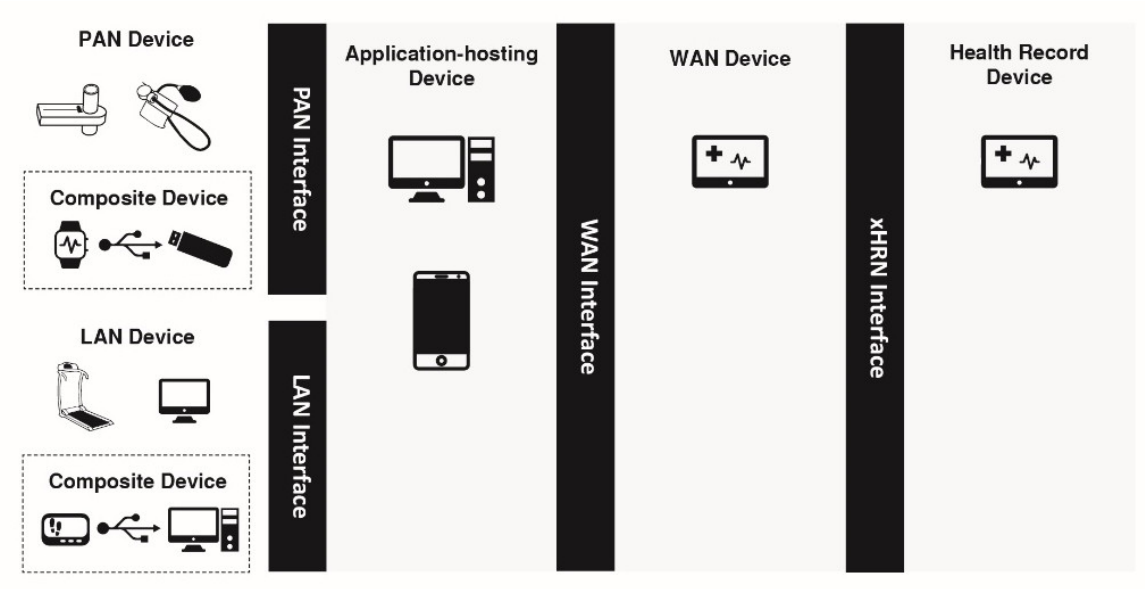

Figure 9. The Continua end-to-end reference architecture (Adapted from Carroll et al. [52]).

The LAN interface is used for communication at a particular location or facility and to connect the LAN devices (which could be a sensor or an actuator) to the application-hosting devices. For example, the Zigbee Health Care Profile can be adopted for the transport layer function [45]. The LAN interface lower layer is based on IP technology such as ethernet and Wi-Fi while the upper layer can support the PAN interface upper layer device data model ISO/IEEE 11073-20601 data model.

The WAN interface is used to link the application hosting device to a variety of WAN services such as health care provider, disease management and implant monitoring services. This interface upper layer uses a device data model which is compatible with that of the LAN interface upper layer 
model. An IP-based technology such as the general packet radio services (GPRS), peripherical or centralised networks (EDGE), and digital subscriber line (xDSL) is used for the WAN interface lower layer. The electronic Health Records Network (xHRN) interface is used to establish a link between the WAN device and a health record device for patient-centric data communications such as health reports to enterprise systems. Also, this interface permits various enterprise healthcare entities to exchange a patient's health information securely.

\section{Discussion}

This literature survey reviewed 16 out of an initial 2923 articles. This low number indicates that research in outdoor space automation for remote health monitoring of the elderly demographic in peer-reviewed literature is limited. The extant literature concentrates on home automation, smart home and home health-monitoring technologies, and their applications in health-service management.

The primary focus of this review is to determine the current approaches of integrating sensors and eHealth technologies in outdoor spaces to collect and transfer data from the elderly demographic, who engage with such built landscapes, to appropriate stakeholders. In terms of sensor integration, the literature can be classified into two main approaches:

1. Wearable: in this approach, the individual to be monitored or tracked wears or carries the sensors [21-29,34,36-41]. A common example of this approach is the smart watch that has multimodal sensors and is therefore capable of sensing multiple physiological parameters through the wrist.

2. Environmental: in this approach, the sensors are deployed in the outdoor space environment or on furniture that will be used by the elderly demographic [32].

It is interesting to note that 15 out of the 16 articles used the wearable approach of sensor integration. This is understandable given the prevalence of wearable devices such as smart watches and mobile phones that are commercially available and ready for use. The latter is especially gaining dominance as the platform of choice for health monitoring (in both indoor and outdoor settings) due to its penetration into populations, availability of apps, connectivity and most importantly, data capture at an individual level [43].

The lack of studies using the environmental sensor integration approach is due to the many difficult challenges it poses on the long-term operation of sensors, chief among which are the lack of power source availability and communication impairments induced by harsh outdoor conditions [53]. While the available wireless communication technologies can be adopted for outdoor use, harsh outdoor conditions can cause unreliable transmission of sensor data. Nevertheless, the wearable approach has a major drawback: the acceptance of wearable technology among the elderly is still ambivalent [54] and they can be obtrusive. The latter is especially problematic. Not only do wearable devices require elderly to wear or carry them on a daily basis, they require substantial attention such as regular recharging, synchronizing and uploading of information to the cloud, and performing regular software updates. These tasks may seem insignificant or trivial to younger or tech-savvy individuals, but to many older people, especially those with cognitive impairments, such impositions they can be a substantial burden.

The eHealth technologies used in the literature reflect the past and more recent communication standards that are already in mass adoption. This is not surprising, as it is generally prohibitively expensive to develop dedicated eHealth technologies just for smart therapeutic and rehabilitation outdoor spaces. With the exception of fixed Internet connectivity, the eHealth technologies used in the proposed solutions are mostly wireless because of its many advantages, especially support for mobility, which is critical in application scenarios where the elderly move in outdoor spaces. The communication range determines the manner by which the eHealth technologies are used, namely:

1. Sensor access: this refers to short-range technologies such as ZigBee, IEEE 802.15.4 and Bluetooth, which are used by the sensor to send its data to a gateway. This is referred to as the PAN and LAN interfaces in the Continua end-to-end reference architecture (Figure 9). 
2. Backhaul access: this refers to long-range technologies such as cellular technology or the fixed IP network, which is used by the gateway to send aggregated sensor data to a server. This is referred to as the WAN interface in the Continua end-to-end reference architecture (Figure 9).

The Bluetooth standard recently adopted a low-power version called Bluetooth Low Energy (BLE) which operates differently from Classic Bluetooth [55]. Huang et al. [56] took advantage of BLE's low energy consumption to develop low-power wearable sensors for long-term monitoring of heart rate, blood oxygen, sleep quality and daily walking steps. A similar standard proposed for wireless body area networks is the IEEE 802.15.6. This technology is designed to enable short-range wireless communications of sensors deployed near or inside the human body [53].

Advances in wireless communication technologies are progressing fast, and there are now newer commercially available technologies that can be used to support the deployment and reliable operation of low-power, low data rate sensors in outdoor settings. Communication technologies such as NB-IoT, LoRa and SigFox [57-59], which fall under the new category of Low Power Wide Area Network (LPWAN), can support a large number of devices within an area, provide long range (10$40 \mathrm{~km}$ in rural areas and 1-5 km in urban areas) and operate on batteries for 10 years or more. Meanwhile, the emerging 5th Generation (5G) cellular network supports machine-to-machine (M2M) type communication for ubiquitous coverage and ultra-long battery life [60].

The smart therapeutic and rehabilitation outdoor space is envisaged to be a part of a bigger ecosystem that aims to deliver better health and wellness outcomes for the elderly. Achieving this aim requires the integration of data collected from the wearable and environmental sensors to the bigger eHealth system. One of the greatest challenges of information eHealth technology in health care delivery is interoperability [61]. Interoperability is defined as the ability of two or more systems, networks, components, applications and devices to exchange information externally and readily use the information that has been exchanged effectively and securely [62-73]. The ultimate goal of interoperability is to enable a seamless bidirectional flow of data (information) between various disparate devices over a network [51].

The need to share and exchange data (information) amongst devices from various vendors and connection of different databases to the internet now make interoperability a key issue that must be addressed to ensure a successful leverage of information technology in health care delivery [62]. Theoretically this can be easily achieved by extensive collaborative efforts from various stakeholders such as manufacturers, system architects, engineers, research and development centres [64]. The eHealth European Interoperability Framework (eEIF) can be useful in deciding the core competencies required for the different activities in interoperability projects. This project identified the various stakeholders who can be involved in different levels of interoperability and confirmed the need for collaborative efforts on various organisational levels with different levels of expertise [4,61].

In order to ensure a seamless deployment of assistive and eHealth technologies for remote monitoring of the elderly demographic with complex needs, there is an urgent research need to deal with the following technical constraints:

- Technology application considering the dynamic and often harsh outdoor environmental conditions, which could hamper availability and accessibility of wireless network services. Research is required to investigate the performance of various eHealth technologies in outdoor spaces for telehealth monitoring. Metrics that can be used to characterize performance include reliability, throughput, latency and security.

- Deployment of interoperable medical devices to ensure devices from various manufactures can be integrated seamlessly in outdoor environments. This means research efforts need to be geared towards developing standards and protocols for heterogeneous devices to communicate in a plug-and-play fashion. Also, there is a need to ensure backward compatibility with existing legacy devices to operate easily with new medical devices.

- Design, development and deployment of technology to manage user mobility and ensure a more accurate localization of older persons in case of emergency. Research related to the human body (e.g., design of wireless PAN) needs to consider a realistic abstraction of the inter-body channel variations which happens as a result of human mobility behaviours [74]. Also, research is 
currently required to design and manufacture power or battery sources with higher efficiency and enhanced battery life for assistive technologies used in a landscape-built environment.

- Data security and confidentiality research is required to avoid unauthorized access to private health information system. The integration of Cloud computing and IoT-based health architectures opens a new research area especially in data security.

\section{Conclusions}

Recent progress in ICT, sensor networks, control engineering, wired and wireless home networking has led to a significant advancement in home automation for remote health monitoring; however, less research has been undertaken on outdoor environments. This review provides an insight about the use of assistive technologies for older persons in smart therapeutic and rehabilitation outdoor spaces. The complexity of the elderly demographic health profiles and the need for an increase in the use of outdoor spaces for cost-effective maintenance and improvement in health and well-being demands greater sophistication in equipment and technologies. For the elderly, it is particularly important to consider person-centeredness, usability and accessibility of eHealth technologies to ensure that meaningful data is captured and feedback is provided so as to avoid technology adoption resistance.

This technology requires a smart interactive platform for integrating collected biometric data via sensor networks which can be shared with health services through eHealth technologies. Reliable eHealth technologies and interfaces are pivotal in ensuring that robust, timely, secure and valid biometrics from the interaction with outdoor landscapes and equipment are exchanged between health centres and patients in such spaces. Biometric data and technology are two critical factors needed to enhance evidence-based decision making, monitoring, evaluation and clinical audit for older people in outdoor spaces.

It is also crucial that new technologies from various vendors can interoperate in such a way that ensures easy and efficient modifications. In addition, apart from being user-friendly, ubiquitous and user-centric, three major considerations for eHealth communication architectures in outdoor spaces are reliability, security and accuracy. Other important factors to be considered in building such smart spaces for older persons are ambient intelligence, computational methods, and effective measurement and communication technologies. Furthermore, smart therapeutic landscapes require onsite remote monitoring tools, a mechanism to trigger assistive technologies for emergency cases and localization of affected older persons.

In general, the level of technology uptake and readiness for smart outdoor spaces is still developing and is currently outpaced by the growth of elderly fitness zones in public spaces. This article will be of value to policy makers, landscape designers, ICT/eHealth engineers, industry partners, medical professionals and other stakeholders affected by age-related loss of function, impairment and disability.

Author Contributions: Conceptualization, B.M. and J.M.; methodology, B.M., J.M., A.G. and A.V.; software, A.G. and A.V.; validation, A.G. and A.V.; formal analysis, B.M., J.M. and A.V.; investigation A.V.; data curation, B.M., J.M., A.G. and A.V.; writing - original draft preparation, B.M. and J.M.; writing - review and editing, B.M., J.M. and A.V.; visualization, B.M.; project administration, J.M. All authors have read and agreed to the published version of the manuscript.

Funding: This research was funded by Victoria University of Wellington Foundation, Digital Innovation Fund, grant number FN00215.

Conflicts of Interest: The authors declare no conflict of interest.

\section{References}

1. World Health Organization [WHO]. Ageing and Health. Available online: http://www.who.int/mediacentre/factsheets/fs404/en/ (accessed on 20 November 2019).

2. World Health Organization [WHO]. National Institute on Aging: Global Health and Aging. Available online: http://www.who.int/ageing/publications/global_health.pdf (accessed on 10 February 2020). 
3. Lohr, K.N.; Field, M.J. Guidelines for Clinical Practice: From Development to Use; National Academies Press: Washington, DC, USA, 1992; pp. 45-64.

4. Nee, O.; Hein, A.; Gorath, T.; Lsmann, N.H.; Laleci, G.B.; Yuksel, M.; Ludwig, R. SAPHIRE: Intelligent healthcare monitoring based on semantic interoperability platform: Pilot applications. IET Commun. 2008, 2, 192-201.

5. Ministry of Health. New Zealand Health Strategy: Future Direction. Available online: https://www.health.govt.nz/system/files/documents/publications/new-zealand-health-strategyfuturedirection-2016-apr16.pdf (accessed on 3 December 2019).

6. Merilampi, S.; Sirkka, A. Introduction to Smart eHealth and eCare Technologies; CRC Press: London, UK, 2016; pp. 3-84.

7. Scholz, N. European Parliamentary Research Service: eHealth-Technology for Health. Available online: http://www.europarl.europa.eu/thinktank/en/document.html?reference=EPRS_BRI(2015)551324 (accessed on 29 November 2019).

8. Iakovidis, I.; Le Dour, O.; Karp, P. Biomedical Engineering and eHealth min Europe-Outcomes and Challenges of Past and Current EU Research Programs. IEEE Eng. Med. Biol. Mag. 2007, 26, $26-28$.

9. Franco, M.R.; Tong, A.; Howard, K.; Sherrington, C.; Ferreira, P.H.; Pinto, R.Z.; Ferreira, M.L. Older people's perspectives on participation in physical activity: A systematic review and thematic synthesis of qualitative literature. Br. J. Sports Med. 2015, 49, 1268-1276.

10. Cranney, L.; Phongsavan, P.; Kariuki, M.; Stride, V.; Scott, A.; Hua, M.; Bauman, A. Impact of an outdoor gym on park users' physical activity: A natural experiment. Health Place 2016, 37, 26-34.

11. Doukas, C.; Metsis, V.; Becker, E.; Le, Z.; Makedon, F.; Maglogiannis, I. Digital cities of the future: Extending @home assistive technologies for the elderly and the disabled. Telemat. Inform. 2011, 28, 176-190.

12. Kershaw, C.; Lim, J.; McIntosh, J.; Cornwall, J.; Marques, B. Developing Resilience, Independence and Wellbeing in Older Adults through Interactive Outdoor Spaces. In Proceedings of the Passive Low Energy Architecture (PLEA) Conference, Edinburgh, Scotland, 2-5 July 2017; Luisa B., Susan R., Fergus N., Eds.; NCEUB: Edinburgh, UK, 2017; pp. 4676-4685.

13. McCormack, G.R.; Rock, M.; Swanson, K.; Burton, L.; Massolo, A. Physical activity patterns in urban neighbourhood parks: Insights from a multiple case study. BMC Public Health 2014, 14, 962.

14. Kershaw, C.; Cornwall, J.; McIntosh, J.; Marques, B. Therapeutic landscape design for older persons health and well-being. In Proceedings of the Third International Conference of Changing Cities, Spatial Design, Landscape and Socio-Economic Dimensions, Syros, Greece, 26-30 June 2017; Aspa G., Ed.; Grafima Publications: Thessaly, Greece, 2017; pp. 1163-1170.

15. Kershaw, C.; McIntosh, J.; Marques, B.; Cornwall, J.; Stoner, L.; Wood, P. A potential role for outdoor, interactive spaces as a healthcare intervention for older persons. Perspect. Public Health 2017, 137, $212-213$.

16. Moher, D.; Liberati, A.; Tetzlaff, J.; Altman, D.G. Preferred reporting items for systematic reviews and metaanalyses: The PRISMA statement. Ann. Intern. Med. 2009, 151, 264-269.

17. Kaklauskas, A.; Abraham, A.; Dzemyda, G.; Raslanas, S.; Seniut, M.; Ubarte, I.; Kurasova, O.; BinkyteVeliene; A.; Cerkauskas, J. Emotional, affective and biometrical states analytics of a built environment. Eng. Appl. Artif. Intell. 2020, 91, 103621.

18. Eysenbach, G. What is e-health? J. Med. Internet Res. 2001, 3, e20.

19. Beauvais, B.S.; Rialle, V.; Sablier, J. Myvigi: An android application to detect fall and wandering. In Proceedings of the Sixth International Conference on Mobile Ubiquitous Computing, Systems, Services and Technologies, Barcelona, Spain, 23-28 September 2012; UBICOMM: Barcelona, Spain; pp. 156-160.

20. Sposaro, F.; Danielson, J.; Tyson, G. iWander: An Android application for dementia patients. In Proceedings of the Engineering in Medicine and Biology Society (EMBC), Buenos Aires, Argentina, 31 August-4 September 2010; IEEE: New Jersey, NJ, USA; pp. 3875-3878.

21. Foxlin, E. Pedestrian tracking with shoe-mounted inertial sensors. IEEE Comput. Graph. Appl. 2005, 25, 3846.

22. Abdel-Aziz, A.A.; Abdel-Salam, H.; El-Sayad, Z. The role of ICTs in creating the new social public place of the digital era. Alex. Eng. J. 2016, 55, 487-493.

23. Lin, C.C.; Chiu, M.J.; Hsiao, C.C.; Lee, R.G.; Tsai, Y.S. Wireless Health Care Service System for Elderly with Dementia. IEEE Trans. Inform. Technol. Biomed. 2006, 10, 696-704. 
24. Lin, C.C.; Lin, P.Y.; Lu, P.K.; Hsieh, G.Y.; Lee, W.L.; Lee, R.G. A Healthcare Integration System for Disease Assessment and Safety Monitoring of Dementia Patients. IEEE Trans. Inform. Technol. Biomed. 2008, 12, 579586.

25. Rainham, D.; Krewski, D.; McDowell, I.; Sawada, M.; Liekens, B. Development of a wearable global positioning system for place and health research. Int. J. Health Geograph. 2008, 7, 59.

26. Oswald, F.; Wahl, H.-W.; Voss, E.; Schilling, O.; Freytag, T.; Auslander, G.; Shoval, N.; Heinik, J.; Landau, R. The Use of Tracking Technologies for the Analysis of Outdoor Mobility in the Face of Dementia: First Steps into a Project and Some Illustrative Findings from Germany. J. Hous. Elder. 2010, 24, 55-73.

27. Zheng, H.; Nugent, C.; McCullagh, P.; Huang, Y.; Zhang, S.; Burns, W.; Davies, R.; Black, N.; Wright, P.; Mawson, S.; et al. Smart self-management: Assistive technology to support people with chronic disease. J. Telemed. Telecare 2010, 16, 224-227.

28. Boulos, M.N.K.; Wheeler, S.; Tavares, C.; Jones, R. How smartphones are changing the face of mobile and participatory healthcare: An overview, with example from eCAALYX. Biomed. Eng. Online 2011, 10, 24.

29. Worringham, C.; Rojek, A.; Stewart, I. Development and Feasibility of a Smartphone, ECG and GPS Based System for Remotely Monitoring Exercise in Cardiac Rehabilitation. PLoS ONE 2011, 6, e14669.

30. Shanin, F.; Das, H.A.; Krishnan, G.A.; Neha, L.S.; Thaha, N.; Aneesh, R.P.; Embrandiri, S.; Jayakrishan, S. Portable and centralised e-health record system for patient monitoring using internet of things (IoT). In Proceedings of the International CET Conference on Control, Communication, and Computing (IC4), Thiruvananthapuram, India, 5-7 July 2018; IEEE: New Jersey, NJ, USA; pp. 165-170.

31. Blaya, J.A.; Fraser, H.S.; Holt, B. E-health technologies show promise in developing countries. Health Aff. 2010, 29, 244-251.

32. Heikkilä, T.; Strömmer, E.; Kivikunnas, S.; Järviluoma, M.; Korkalainen, M.; Kyllönen, V.; Sarjanoja, E.M.; Peltomaa, I. Low intrusive Ehealth monitoring: Human posture and activity level detection with an intelligent furniture network. IEEE Wirel. Commun. 2013, 20, 57-63.

33. Schwickert, L.; Becker, C.; Lindemann, U.; Maréchal, C.; Bourke, A.; Chiari, L.; Helbostad, J.L.; Zijlstra, W.; Aminian, K.; Todd, C.; et al. Fall detection with body-worn sensors. Z. Gerontol. Geriatr. 2013, 46, 706-719.

34. Yared, R.; Mallat, H.K.; Abdulrazak, B. Ambient technology to support elderly people in outdoor risk situations. In Proceedings of the International Conference on Information and Communication Technologies for Ageing Well and e-Health, Lisbon, Portugal, 20-22 May 2015; Springer: Cham, Switzerland; pp. 35-56.

35. Islam, S.R.; Kwak, D.; Kabir, M.H.; Hossain, M.; Kwak, K.S. The internet of things for health care: A comprehensive survey. IEEE Access 2015, 3, 678-708.

36. Wang, L.H.; Hsiao, Y.M.; Xie, X.Q.; Lee, S.Y. An outdoor intelligent healthcare monitoring device for the elderly. IEEE Trans. Consum. Electron. 2016, 62, 128-135.

37. Hossain, M.S.; Muhammad, G. Cloud-assisted Industrial Internet of Things (IIoT) - Enabled framework for health monitoring. Comput. Netw. 2016, 101, 192-202.

38. Almeida, A.; Fiore, A.; Mainetti, L.; Mulero, R.; Patrono, L.; Rametta, P. An IoT-Aware Architecture for Collecting and Managing Data Related to Elderly Behavior. Wirel. Commun. Mob. Comput. 2017, 2017, 5051915.

39. Hu, J.-X.; Chen, C.-L.; Fan, C.-L.; Wang, K.-H. An Intelligent and Secure Health Monitoring Scheme Using IoT Sensor Based on Cloud Computing. J. Sens. 2017, 2017, 3734764.

40. Garcia, A.C.B.; Vivacqua, A.S.; Sánchez-Pi, N.; Martí, L.; Molina, J.M. Crowd-Based Ambient Assisted Living to Monitor the Elderly's Health Outdoors. IEEE Softw. 2017, 34, 53-57.

41. Chan, M.; Campo, E.; Bourennane, W.; Estève, D. Connectivity for the indoor and outdoor elderly people safety management: An example from our current project. In Proceedings of the European Symposium on Biomedical Engineering (ESBME 2010), Kuala Lumpur, Malaysia, 30 November-2 December 2010; pp. 14.

42. Boulos, M.N.K.; Brewer, A.C.; Karimkhani, C.; Buller, D.B.; Dellavalle, R.P. Mobile medical and health apps: State of the art, concerns, regulatory control and certification. Online J. Public Health Inform. 2014, 5, 229.

43. Davis, T.L.; DiClemente, R.; Prietula, M. Taking mHealth Forward: Examining the Core Characteristics. JMIR mHealth uHealth 2016, 4, e97.

44. Coughlan, J.; Manduchi, R. Functional assessment of a camera phone-based wayfinding system operated by blind and visually impaired users. Int. J. Artif. Intell. Tools 2009, 18, 379-397. 
45. ETSI. eHEALTH; Architecture; Analysis of User Service Models, Technologies and Applications Supporting eHealth. Available online: https://www.etsi.org/deliver/etsi_tr/102700_102799/102764/01.01.01_60/tr_102764v010101p.pdf (accessed on 29 January 2020).

46. Piette, J.D.; Lun, K.C.; Moura, L.A., Jr.; Fraser, H.S.; Mechael, P.N.; Powell, J.; Khoja, S.R. Impacts of e-health on the outcomes of care in low-and middle-income countries: Where do we go from here? Bull. World Health Organ. 2012, 90, 365-372.

47. Wiljer, D.; Urowitz, S.; Apatu, E.; DeLenardo, C.; Eysenbach, G.; Harth, T.; Pai, H.; Leonard, K.; Canadian Committee for Patient Accessible Health Records (CCPAEHR). Patient accessible electronic health records: Exploring recommendations for successful implementation strategies. J. Med. Internet Res. 2008, 10, e34.

48. Pagliari, C.; Detmer, D.; Singleton, P. Potential of electronic personal health records. BMJ Br. Med. J. 2007, 335, 330.

49. Chehri, A.; Mouftah, H.; Jeon, G. A Smart Network Architecture for eHealth Applications. In Intelligent Interactive Multimedia Systems and Services; Tsihrintzis, G.A., Damiani, E., Virvou, M., Howlett, R.J., Jain, L.C., Eds.; Springer: Berlin, Germany, 2010; pp. 157-166.

50. Wartena, F.; Muskens, J.; Schmitt, L.; Petkovic, M. Continua: The reference architecture of a personal telehealth ecosystem. In Proceedings of the 12th IEEE International Conference on e-Health Networking Applications and Services (Healthcom 2010), Lyon, France, 1-3 July 2010; IEEE: New Jersey, NJ, USA, 2010; pp. 399-404.

51. Moorman, B. Medical Device Interoperability: Overview of Key Initiatives. Biomed. Instrum. Technol. 2010, 44, 132-138.

52. Carroll, R.; Cnossen, R.; Schnell, M.; Simons, D. Continua: An Interoperable Personal Healthcare Ecosystem. IEEE Pervasive Comput. 2007, 6, 90-94.

53. Kartsakli, E.; Lalos, A.S.; Antonopoulos, A.; Tennina, S.; Renzo, M.D.; Alonso, L.; Verikoukis, C. A Survey on M2M Systems for mHealth: A Wireless Communications Perspective. Sensors 2014, 14, 18009-18052.

54. Wark, T.; Hu, W.; Corke, P.; Hodge, J.; Keto, A.; Mackey, B.; Foley, G.; Sikka, P.; Brunig, M. Springbrook: Challenges in developing a long-term, rainforest wireless sensor network. In Proceedings of the 2008 International Conference on Intelligent Sensors, Sensor Networks and Information Processing, Sydney, Australia, 15-18 December 2008; IEEE: New Jersey, NJ, USA; pp. 599-604.

55. Tedesco, S.; Barton, J.; O’Flynn, B. A review of activity trackers for senior citizens: Research perspectives, commercial landscape and the role of the insurance industry. Sensors 2017, 17, 1277.

56. Huang, P.C.; Lin, C.C.; Wang, Y.H.; Hsieh, H.J. Development of Health Care System Based on Wearable Devices. In Proceedings of the 2019 Prognostics and System Health Management Conference (PHM-Paris), Paris, France, 2-5 May 2019; IEEE: New Jersey, NJ, USA; pp. 249-252.

57. Yared, R.; Abou Jaoude, C.; Demerjian, J. Smart-phone based system to monitor walking activity: MHealth solution. In Proceedings of the 2018 IEEE Middle East and North Africa Communications Conference (MENACOMM), Jounieh, Lebanon, 18-20 April 2018; IEEE: New Jersey, NJ, USA; pp. 1-5.

58. Mekki, K.; Bajic, E.; Chaxel, F.; Meyer, F. A comparative study of LPWAN technologies for large-scale IoT deployment. ICT Express 2019, 5, 1-7.

59. Gaddam, A.; Wilkin, T.; Angelova, M.; Valera, A.; McIntosh, J.; Marques, B. Design \& Development of IoT Based Rehabilitation Outdoor Landscape for Gait Phase Recognition. In Proceedings of the 2019 13th International Conference on Sensing Technology (ICST) 2019, Sydney, Australia, 2-4 December 2019; IEEE: New Jersey, NJ, USA; pp. 1-7.

60. Mehmood, Y.; Haider, N.; Imran, M.; Timm-Giel, A.; Guizani, M. M2M communications in 5G: State-ofthe-art architecture, recent advances, and research challenges. IEEE Commun. Mag. 2017, 55, 194-201.

61. European Commission. eHealth Network: Refined eHealth European Interoperability Framework. Available online: https://ec.europa.eu/health//sites/health/files/ehealth/docs/ev_20151123_co03_en.pdf (accessed on 10 December 2019).

62. Nijeweme-D’Hollosy, W.O.; Velsen, L.V.; Huygens, M.; Hermens, H. Requirements for and Barriers towards Interoperable eHealth Technology in Primary Care. IEEE Internet Comput. 2015, 19, 10-19.

63. Rezaei, R.; Chiew, T.K.; Lee, S.P.; Shams Aliee, Z. Interoperability evaluation models: A systematic review. Comput. Ind. 2014, 65, 1-23. 
64. Photovoltaics, D.G.; Storage, E. IEEE Guide for Smart Grid Interoperability of Energy Technology and Information Technology Operation with the Electric Power System (EPS), End-Use Applications, and Loads; IEEE: New York, NY, USA, 2011; pp. 1-126, doi:10.1109/IEEESTD.2011.6018239.

65. Sheikh, A.; Sood, H.S.; Bates, D.W. Leveraging health information technology to achieve the "triple aim" of healthcare reform. J. Am. Med. Inform. Assoc. 2015, 22, 849-856.

66. Zutshi, A.; Grilo, A.; Jardim-Goncalves, R. The business interoperability quotient measurement model. Comput. Ind. 2012, 63, 389-404.

67. Naudet, Y.; Latour, T.; Guedria, W.; Chen, D. Towards a systemic formalisation of interoperability. Comput. Ind. 2010, 61, 176-185.

68. Munk, S. An analysis of basic interoperability related terms, system of interoperability types. Acad. Appl. Res. Mil. Sci. 2002, 2002, 117-132.

69. Novakouski, M.; Lewis, G. Interoperability in the e-Government Context (CMU/SEI-2011-TN-014). Available online: http://resources.sei.cmu.edu/library/asset-view.cfm?AssetID=9869 (accessed on 2 February 2020).

70. Veer, H.; Wiles, A. Achieving Technical Interoperability-the ETSI Approach, European Telecommunications Standards Institute. Available online: https://www.etsi.org/images/files/ETSIWhitePapers/IOP\%20whitepaper\%20Edition\%203\%20final.pdf (accessed on 10 November 2019).

71. Guijarro, L. Semantic interoperability in eGovernment initiatives. Comput. Stand. Interfaces 2009, 31, 174180.

72. Charalabidis, Y.; Panetto, H.; Loukis, E.; Mertins, K. Interoperability approaches for enterprises and administrations worldwide. Electron. J. e-Commer. Tools Appl. 2008, 2, 1-10.

73. Scott, P. Meeting the challenges of healthcare interoperability. Healthc. IT Manag. 2009, 4, 24-25.

74. Alam, M.; Hamida, E. Surveying Wearable Human Assistive Technology for Life and Safety Critical Applications: Standards, Challenges and Opportunities. Sensors 2014, 14, 9153-9209.

Publisher's Note: MDPI stays neutral with regard to jurisdictional claims in published maps and institutional affiliations.

(C) 2020 by the authors. Licensee MDPI, Basel, Switzerland. This article is an open access article distributed under the terms and conditions of the Creative Commons Attribution (CC BY) license (http://creativecommons.org/licenses/by/4.0/). 